Impact of a demanding movement on decision-making

9

\title{
Humans sacrifice decision-making for action execution when a demanding control of movement is required
}

\author{
Amélie J. REYNAUD, Clara SALERI LUNAZZI, David THURA \\ Lyon Neuroscience Research Center - Impact team \\ Inserm U1028 - CNRS UMR5225 - Lyon University
}

Running head

Impact of a demanding movement on decision-making

Corresponding author information

David Thura

Lyon Neuroscience Research Center - Impact team Inserm U1028 - CNRS UMR5225 - Lyon University 16 avenue du Doyen Lépine, 69675 Bron, France

Phone : +33472913414

E-mail: david.thura@inserm.fr

Manuscript information

Total word count: $~ 10700 ; 40$ pages; 8 figures

Conflict of interest

The authors declare no competing financial interests

Funding

This work was supported by a CNRS/Inserm ATIP/Avenir grant and an Inserm young investigator fellowship to DT 
Impact of a demanding movement on decision-making

\section{ABSTRACT}

33 A growing body of evidence suggests that decision-making and action execution are governed by 34 partly overlapping operating principles. Especially, previous work proposed that a shared 35 decision urgency/movement vigor signal, possibly computed in the basal ganglia, coordinates 36 both deliberation and movement durations in a way that maximizes the reward rate. Recent data 37 support one aspect of this hypothesis, indicating that the urgency level at which a decision is 38 made influences the vigor of the movement produced to express this choice. Here we 39 investigated whether conversely, the motor context in which a movement is executed determines 40 decision speed and accuracy. Twenty human subjects performed a probabilistic decision task in 41 which perceptual choices were expressed by reaching movements toward targets whose size and

42 distance from a starting position varied in distinct blocks of trials. We found strong evidence for 43 an influence of the motor context on most of the subjects' decision policy but contrary to the 44 predictions of the "shared regulation" hypothesis, we observed that slow movements executed in 45 the most demanding motor blocks in terms of accuracy were often preceded by faster and less 46 accurate decisions compared to blocks of trials in which big targets allowed expression of

47 choices with fast and inaccurate movements. These results suggest that decision-making and 48 motor control are not regulated by one unique "invigoration" signal determining both decision 49 urgency and action vigor, but more likely by independent, yet interacting, decision urgency and 50 movement vigor signals.

53 Recent hypotheses propose that choices and movements share optimization principles derived 54 from economy, possibly implemented by one unique context-dependent regulation signal 55 determining both processes speed. In the present behavioral study conducted on human subjects, 
bioRxiv preprint doi: https://doi.org/10.1101/2020.04.08.028936; this version posted July 7, 2020. The copyright holder for this preprint (which was not certified by peer review) is the author/funder, who has granted bioRxiv a license to display the preprint in perpetuity. It is made available under aCC-BY-NC-ND 4.0 International license.

Impact of a demanding movement on decision-making

56 we demonstrate that action properties indeed influence perceptual decision-making, but that

57 decision duration and action vigor are actually independently set depending on the difficulty of

58 the movement executed to report a choice.

59

60

\section{KEYWORDS}

61

Decision-making, Reaching, Urgency, Speed-accuracy trade-off, Human 
Impact of a demanding movement on decision-making

\section{INTRODUCTION}

63 Animals, including humans, are faced with decisions about actions on a daily basis, and they

64 behave to seek rewards while avoiding punishments and minimizing energy expenditure. Because

65 the evaluation of reward, risk, and effort governs our action choices, investigating how the brain

66 processes these variables is critical to improve our understanding of adapted or dysfunctional

67 goal-directed behavior.

68 Importantly, the subjective value of a given activity is not only limited to its related reward, risks,

69 and efforts. It also depends on the amount of time invested in it, as time strongly discounts the

70 value of rewards (Myerson and Green, 1995). Therefore, what is ultimately most adaptive is to

71 choose options that maximize one's global reward rate (Bogacz et al., 2010; Balci et al., 2011),

72 which occurs when the decision and action processes are sufficiently accurate but not overly

73 effortful and time-consuming. As a consequence, nearly all decision scenarios present decision-

74 makers with speed-accuracy-effort trade-offs during both decision-making and action execution,

75 and the brain must control both processes to maximize the rate of reward.

76 Because trade-offs during decision and action have been typically studied in isolation,

77 mechanisms allowing a coordinated maximization of reward rate are still elusive. Recent

78 promising advances suggest, however, that motor control and choices, including economic ones,

79 are governed by partly overlapping optimization principles (Shadmehr et al., 2010, 2019; Haith et

80 al., 2012; Choi et al., 2014; Yoon et al., 2018; Carland et al., 2019). First, human and non-human

81 primates move faster and with a shorter reaction time toward items that they value more

82 (Kawagoe et al., 1998; Summerside et al., 2018; Revol et al., 2019). Second, humans take motor

83 costs into account during both motor (Cos et al., 2011, 2012, 2014; Morel et al., 2017) and non-

84 motor (Burk et al., 2014; Marcos et al., 2015; Diamond et al., 2017; Hagura et al., 2017) 
Impact of a demanding movement on decision-making

85 decisions and effortful reaches impose a cost for decision-making similar to cost functions in

86 motor control (Wickler et al., 2000; Shadmehr et al., 2016; Morel et al., 2017; Reppert et al.,

87 2018). Finally, in the foraging paradigm where one makes decisions regarding how long to stay

88 and accumulate reward from one patch, and then moves with certain speed to another patch, the

89 goods collection duration and the vigor (movement speed and duration) with which human

90 subjects move from one reward site to another are governed by a mechanism allowing to

91 maximize the overall capture rate (Yoon et al., 2018).

92 In line with this shared optimization hypothesis, we and others have proposed that control of

93 urgency is critical for reward rate maximization during decision-making between actions

94 (Ditterich, 2006; Churchland et al., 2008; Standage et al., 2011; Thura et al., 2012; Malhotra et

95 al., 2017, 2018). Urgency is a context-dependent, motor-related signal that grows over the time

96 course of deliberation, pushing the decision-related neural activity toward the commitment

97 threshold (Thura and Cisek, 2014; Kira et al., 2015; Murphy et al., 2016; Steinemann et al.,

98 2018). Remarkably, we demonstrated in a changing evidence decision task that urgency level at

99 decision time strongly influences speed and duration of the following motor commands: early

100 decisions, usually made based on strong sensory evidence but low urgency, were followed by

101 long movements (in terms of duration) whereas late decisions, relying on weak sensory evidence

102 but strong urgency, were followed by faster movements. Then, when subjects were encouraged to

103 make faster and less accurate decisions in distinct blocks of trials, movements were faster

104 compared to blocks encouraging slow and accurate choices. These results imply that a shared

105 invigoration signal, possibly computed in the basal ganglia, coordinates the unified adaptation of

106 the speed-accuracy trade-off during both decision-making and action execution in order to 
Impact of a demanding movement on decision-making

107 control the rate of reward (Thura et al., 2014; Thura and Cisek, 2016, 2017; Cisek and Thura,

108 2018; Thura, 2020).

109 We proposed a model of this hypothetical mechanism, labeled the "shared regulation" hypothesis

110 (Figure 1A, Thura et al., 2014). In this model, speed-accuracy trade-offs for deciding and acting

111 are influenced by a shared decision urgency/movement vigor signal. As a consequence, the

112 context-dependent urgency level at which a decision is made should determine the vigor

113 (duration and speed scaled by amplitude) of movements produced to express this choice and

114 conversely, the context-dependent vigor of movements executed to express a choice should

115 predict the level of urgency with which that choice is made. Recent behavioral and

116 neurophysiological data collected in both trained monkeys and naïve humans strongly support the

117 former prediction (Thura et al., 2014; Thura and Cisek, 2016; Thura, 2020). The latter prediction,

118 namely whether or not the fastest choices are made in motor contexts encouraging the most

119 vigorous movements (Figure 1B), remains, however, to be tested.

120 To this aim, we conducted an experiment in which human subjects performed a probabilistic

121 decision task in which perceptual choices were expressed by reaching movements toward targets

122 whose size and distance from the starting point varied across blocks of trials, allowing us to

123 assess the effects of the motor context on subjects' decision policy. In the present work, the speed

124 and duration of the movements are considered as indicators of action vigor and the movement

125 speed-accuracy trade-off are used to modulate this vigor.

126 
Impact of a demanding movement on decision-making

129 Twenty-three healthy, human subjects (ages: 18-41; 17 females; 21 right-handed) participated in

130 this study. All gave their consent orally before starting the experiment. The ethics committee of

131 Inserm (IRB00003888) approved the protocol on March $19^{\text {th }}, 2019$. Each participant was asked to

132 perform two experimental sessions. They received monetary compensation ( $20 €$ per completed

133 session) for participating in this study. Among them, twenty (ages: $20-41$; 16 females; 18 right-

134 handed) completed at least two sessions and have thus been included in the present dataset.

\section{Dataset}

136 The decision and motor behaviors of most of the subjects (17/20) have been described in a recent

137 publication aimed to report the effect of decision strategy on movement properties in human

138 subjects (Thura, 2020). This analysis showed that according to the shared regulation hypothesis,

139 the urgency level at the time of decision commitment strongly influences movement kinematics,

140 with urgency-based decisions leading to vigorous movements. In the present paper, we analyzed

141 data of the same subjects along with data from 3 additional ones, but we grouped trials depending

142 on movement constraints (target size/movement amplitude configurations, see below), allowing

143 us to test on the same subjects the reverse side of the shared regulation hypothesis, i.e. the effects

144 of motor context on decision policy.

145 Setup

146 The subjects sat in an armchair made planar reaching movements using a handle held in their

147 dominant hand (Figure 2A). A digitizing tablet (GTCO CalComp) continuously recorded the

148 handle horizontal and vertical positions (100 Hz with $0.013 \mathrm{~cm}$ accuracy). Target stimuli and

149 cursor feedback were projected by a DELL P2219H LCD monitor (60 Hz refresh rate) onto a

150 half-silvered mirror suspended $26 \mathrm{~cm}$ above and parallel to the digitizer plane, creating the

151 illusion that targets floated on the plane of the tablet. Unconstrained eye movements and pupil 
Impact of a demanding movement on decision-making

152 area of a subset of subjects were recorded using an infrared camera (ISCAN, sampling rate of 120

$153 \mathrm{~Hz}$, data not shown).

154 Tasks

155 The subjects performed a modified version of the tokens task (Figure 2B, see Cisek et al., 2009

156 for the original version). They were faced with a visual display consisting of three blue circles

157 (1.5 cm radius) placed horizontally at a distance of $6 \mathrm{~cm}$ of each other (the "decision" stimuli). In

158 the central blue circle, 15 small tokens were randomly arranged. Positioned $12 \mathrm{~cm}$ below, three

159 black circles, organized horizontally as well defined the "movement" stimuli. While the central

160 black circle radius was kept constant at $0.75 \mathrm{~cm}$, the size of the two lateral black circles and their

161 distance from the central circle could vary, set to either 0.75 (small) or $1.5 \mathrm{~cm}$ (big) of radius, and

162 either 6 (short) or $12 \mathrm{~cm}$ (long) of distance from the central circle, in distinct blocks of trials. This

163 design allowed us to define four motor blocks depending on the size/distance combination of the

164 two targets: "small/short", "small/long", "big/short" and "big/long” (Figure 2C).

165 A trial was initiated when the subject moved and hold the handle into the small black central

166 circle (starting position) for 500ms. Tokens then started to jump, one by one, every $200 \mathrm{~ms}$ in one

167 of the two possible lateral blue circles. The subjects' task was to decide which of the two lateral

168 blue circles would receive the majority of the tokens at the end of the trial. They reported their

169 decisions by moving the handle into the lateral black circle corresponding to the side of the

170 chosen blue circle. Importantly, subjects were allowed to make and report their choice at any time

171 between the first and the last jump. Arm movement duration could not exceed 800ms,

172 irrespective of the motor block. If a movement exceeds $800 \mathrm{~ms}$ (too slow) or if it reaches the

173 target but fails to stop in it within $800 \mathrm{~ms}$ (inaccurate), the trial is considered as a movement error

174 trial. Once the choice is properly reported, the remaining tokens jumped more quickly to their 
Impact of a demanding movement on decision-making

175 final circles. In separate blocks of trials, this post-decision interval was set to either 20ms ("fast"

176 decision block) or 150ms ("slow" decision block). The acceleration of the remaining tokens

177 implicitly encouraged subjects to decide before all tokens had jumped into their respective lateral

178 circles, to save time and increase their rate of reward. Note that each reaching movement carries a

179 temporal cost with respect to reward rate maximization (see equation 3) because the remaining

180 tokens accelerate only when action is completed. After holding the handle in the target for

$181500 \mathrm{~ms}$, visual feedback about decision success or failure (the chosen decision circle turning

182 either green or red, respectively) was provided after the last token jump. A 1500ms period (the

183 inter-trial interval) preceded the following trial.

184 Before and after the tokens task described above, each subject also performed 100 trials of a

185 delayed reach task (DR task). This task was identical to the tokens task except that there was only

186 one lateral decision circle displayed at the beginning of the trial (either at the right or the left side

187 of the central circle with $50 \%$ probability) and all tokens moved from the central circle to this

188 unique circle at a GO signal occurring after a variable delay (1000 $\pm 150 \mathrm{~ms})$. They executed 2

189 different motor blocks of 25 trials each before the tokens task and the 2 other motor blocks (25

190 trials each) after the tokens task. This DR task was used to estimate the sum of the delays

191 attributable to sensory processing of the stimulus display as well as to response initiation in each

192 motor condition.

\section{Instructions}

194 In a given session, subjects were asked to complete one slow decision block and one fast decision

195 block of the tokens task. To complete a decision block (either fast or slow), subjects had to make

196160 correct choices, indirectly motivating them to optimize successes per unit of time. After the

197 first block was completed, a short break was offered to the subject. Within each decision block, 
Impact of a demanding movement on decision-making

198 the size of the movement targets and their distance from the starting circle, i.e. the motor blocks,

199 were varied every 40 trials. In a session, each motor block was thus performed twice, once in the

200 slow decision block, and once in the fast decision block.

201 Subjects performed two sessions (test-retest design), one a day, and each of them separated by a 202 maximum of 7 days. In session \#1 subjects always started the tokens task in the slow decision 203 block with the following succession of motor blocks: small/short, small/long, big/short, and 204 big/long; followed by the execution of the fast decision block with the same motor blocks order.

205 To prevent any block-related confounding effect, the order of decision and motor blocks 206 presentation was reversed in session \#2. Before the first session, we explicitly described to the 207 subjects the principle of each decision block, specifying that deciding quickly in the fast block 208 was more advantageous in terms of time-saving than in the slow block (because of the larger 209 acceleration of the remaining tokens) but that such hasty behavior could also lead to more 210 erroneous decisions. A short recall was provided before starting the second session. Because 211 subjects were informed that they had to complete a given number of correct responses in each 212 session, they were all aware that they were presented with a speed/accuracy trade-off in this task. 213 A practice period consisting of performing 20 tokens task trials in the slow decision and big/short 214 motor blocks was proposed at the beginning of the first session, mainly allowing subjects to get 215 familiar and comfortable with the manipulation of the handle on the tablet. Among the 23 216 subjects who participated in this study, two have been tested six and seven times. The additional 217 sessions performed by these two subjects are not described in the present report.

\section{Data analysis}

219 All arm movement data were analyzed off-line using MATLAB (MathWorks). Reaching 220 characteristics were assessed using the subjects' movement kinematics. Horizontal and vertical 
Impact of a demanding movement on decision-making

221 position data were first filtered using a tenth-degree polynomial filter and then differentiated to 222 obtain a velocity profile. Onset and offset of movements were determined using a $3.75 \mathrm{~cm} / \mathrm{s}$

223 velocity threshold. Peak velocity was determined as the maximum value between these two

224 events and endpoint error was defined as the Euclidian distance separating the target center from

225 the movement endpoint location. The dispersion of movement end-points is visualized with

226 confidence ellipses representing an iso-contour of the Gaussian distribution, defining the region

227 that contains $95 \%$ of all samples in each condition.

228 We computed at each moment during a trial the success probability $p_{i}(t)$ associated with choosing 229 each target $i$. For a total of 15 tokens, if at a particular moment in time the right target contains $N_{R}$ 230 tokens, whereas the left contains $N_{L}$ tokens, and there are $N_{C}$ tokens remaining in the center, then 231 the probability that the target on the right will ultimately be the correct one (i.e., the success 232 probability of guessing right) is as follows:

$$
p\left(\mathrm{R} \mid N_{R}, N_{L}, N_{C}\right)=\frac{N_{C} !}{2^{N_{C}}} \sum_{k=0}^{\min \left(N_{C}, 7-N_{L}\right)} \frac{1}{k !\left(N_{C}-k\right) !}
$$

233 To characterize the success probability profile of each trial, we calculated this quantity (with 234 respect to either the correct target or the target ultimately chosen by the subject, depending on 235 purposes) for each token jump. To ensure that the difficulty of decisions was homogeneous 236 among subjects and experimental conditions, we controlled the sequence of trials experienced by 237 subjects in each session. Especially, we interspersed among fully random trials (20\% of the trials 238 in which each token is $50 \%$ likely to jump into the right or the left lateral circle) three special 239 types of trials characterized by particular temporal profiles of success probability. Subjects were 240 not told about the existence of these trials. $30 \%$ of trials were so-called "easy" trials, in which 241 tokens tended to move consistently toward one of the circles, quickly driving the success 
Impact of a demanding movement on decision-making

242 probability $p_{i}(t)$ for each toward either 0 or 1 . Another $30 \%$ of trials were "ambiguous", in which

243 the initial token movements were balanced, making the $p_{i}(t)$ function close to 0.5 until later in the

244 trial. The last special trial type was called "misleading" trials (20\%) in which the 2-3 first tokens

245 jumped into the incorrect circle and the remaining ones into the correct circle. In all cases, even

246 when the temporal profile of success probability of a trial was predesigned, the actual correct

247 target was randomly selected on each trial. Importantly, the sequence of trials was designed such

248 as the proportion of each trial type was similar in each decision and motor condition (Figure 2D).

249 To estimate the time at which subjects committed to their choice (decision time, DT) on each trial

250 in the tokens task, we detected the time of movement onset, defining the subject's reaction time

251 (RT) and subtracted from it her/his mean sensory-motor delays (SM) estimated based on her/his

252 reaction times in the same motor block of the delayed reach task performed the same day.

253 Decision duration (DD) was computed as the duration between the DT and the first token jump.

254 Equation 1 was then used to compute for each trial the success probability at the time of the 255 decision (SP).

256 Calculation of subjects' accuracy criterion at decision time relies on the available sensory 257 evidence at that time. Because it is very unlikely that subjects can calculate Equation 1, we 258 computed a simple "first-order" approximation of sensory evidence as the sum of log-likelihood 259 ratios (SumLogLR) of individual token movements as follows (Cisek et al., 2009, page 11567, 260 provides more details on this analysis):

$$
\operatorname{SumLogLR}(n)=\sum_{k=1}^{n} \log \frac{p\left(e_{k} \mid S\right)}{p\left(e_{k} \mid U\right)}
$$

261 where $p\left(e_{k} \mid S\right)$ is the likelihood of a token event $e_{k}$ (a token jumping into either the selected or 262 unselected target) during trials in which the selected target $S$ is correct, and $p\left(e_{k} \mid U\right)$ is its 
Impact of a demanding movement on decision-making

263 likelihood during trials in which the unselected target $U$ is correct. The SumLogLR metric is thus

264 proportional to the difference in the number of tokens that have moved in each circle before the

265 moment of decision. To characterize the decision policy of a given subject in a given block of

266 trials, we binned trials as a function of the total number of tokens that moved before the decision

267 and calculated the average SumLogLR for each bin.

268 To quantify subjects' performance relative to the task objective, i.e. complete a given number of 269 correct decisions, assuming they tried to complete each block as quickly as possible, we first 270 calculated for correct and bad decisions the reward rate (RR), using a local definition (Haith et 271 al., 2012; Thura et al., 2012) which corresponds to the expected number of correct choices per 272 unit of time. This is computed as follows:

$$
R R_{n}=\frac{S P_{n}}{D D_{n}+S M+M D_{n}+R D_{n}+I T I}
$$

273 where $\mathrm{SP}_{n}$ is the probability that the choice made on trial $n$ was correct, $\mathrm{DD}_{n}$ is the time taken to

274 make the decision, SM is the sensorimotor delays (specific to each motor context but constant for 275 a given session), $\mathrm{MD}_{n}$ is the movement duration, $\mathrm{RD}_{n}$ is the duration of the remaining token 276 jumps after the target is reached, and ITI is the inter-trial interval (fixed at 1500ms). Then from 277 the average reward rate computed in each motor block, we calculated the average number of 278 correct choices per minute and deducted from it the time necessary to complete a given number 279 of correct choices in each condition of interest.

280 Comparisons of decision duration, success probability, movement duration, peak velocity, 281 accuracy, and block duration between conditions performed for each subject are statistically 282 tested with Wilcoxon-Mann-Whitney (WMW, two-sided rank-sum) tests. The effect of motor 283 condition on sensory evidence at decision time as a function of decision duration is statistically 
Impact of a demanding movement on decision-making

284 tested with analyses of covariance (ANCOVAs). For these analyses, very fast decisions made

285 before token jump \#4 are discarded. Decisions made before jump \#4 were rare (see Thura, 2020)

286 and success probability homogeneity (if subjects decide before token jump \#4 it is likely because

287 the first three tokens jumped into the same target) at that time makes data exclusion reasonable.

288 The proportion of inadequate movements in small target conditions (small/short and small/long

289 blocks) is statistically compared to the proportion of inadequate movements in big target

290 conditions (big/short and big/long blocks) for each subject with chi-square tests. For all statistical

291 tests, the significance level is set a 0.05 .

\section{RESULTS}

\section{Effect of motor context on motor behavior in the tokens task}

295 As expected, the motor context in which decisions were reported strongly influenced subjects' 296 movement properties and performance. First, we calculated the percentage of trials in which an 297 inadequate movement was performed to express a choice, i.e. a movement exceeding $800 \mathrm{~ms}$ (too 298 slow) or failing to stop and maintain the position in the target within $800 \mathrm{~ms}$ (inaccurate). In the 299 first session, most subjects (18/20) performed significantly more inadequate movements in the 300 small target (small/short and small/long blocks) condition compared to the big target (big/short 301 and big/long blocks) condition (Chi-square tests, $\mathrm{p}<0.05$ ). Movement "error" rates within blocks 302 are the following across the population: small/long target blocks:18.8\% \pm 6.8 ; small/short: $5.5 \%$ $303 \pm 3.1$; big/long: $4.5 \% \pm 2.7$; big/short: $1 \% \pm 1.3$. Despite an overall slight decrease, the same 304 impact of motor constraint was observed on movement error rate during session \#2: 19 out of 20 305 subjects made more inadequate movements in the small target compared to the big target 306 condition (Chi-square tests, $\mathrm{p}<0.05$ ), with the following error rates in each of the four motor 
Impact of a demanding movement on decision-making

307 contexts: small/long target blocks: $16.7 \% \pm 4.5$; small/short: $4.8 \% \pm 1.8$; big/long: $2.35 \% \pm 1.5$;

308 big/short: $1.1 \% \pm 1.3$ ). Figure 3 A shows the dispersion of movement endpoints in one example

309 subject who performed the tokens task in the four motor blocks. In this plot, correct and

310 inadequate (too slow or inaccurate) movements trials are included. Confidence ellipses

311 (containing 95\% of all samples in each condition) largely extend outside of movement targets in

312 small target trials, especially when targets are far from the starting center, whereas they almost

313 entirely fit into movement targets in big target trials.

314 Then, we focused analyses on trials in which an adequate movement was performed to express a

315 choice, irrespective of the outcome of that choice. As expected, reaching movement properties, in

316 terms of velocity peak, duration, and endpoint "error" (the distance between target center and

317 movement offset location) were affected by the motor context in which movements were

318 executed. Figure 3B shows for the same representative subject the reaching velocity profiles in

319 trials sorted as a function of the four motor blocks. Unsurprisingly, movement velocity was

320 largely higher and duration longer in long target (dotted lines) compared to short target trials

321 (solid lines), regardless of the size of the target. The size of the target also modulated movement

322 speed and duration but to a lesser extent. Movements were indeed slightly faster and shorter

323 when executed toward big targets (orange lines) compared to those executed toward small targets

324 (blue lines).

325 This effect of motor context on movement properties was observed on the vast majority of 326 subjects performing either the tokens or the delayed reach (DR) task. To simplify comparisons in 327 the following analyses, we grouped trials depending on (1) target size, defining two conditions, 328 small versus big target conditions, regardless of target distance from the starting circle, and (2) 
Impact of a demanding movement on decision-making

329 target distance from the starting circle, defining two other conditions, short versus long target

330 conditions, regardless of target size.

331 First, most of the subjects reported decisions by making significantly faster (15 out of 20

332 subjects, WMW test, $\mathrm{p}<0.05)$, shorter, in terms of duration (17 out of 20 subjects, WMW test,

$333 \mathrm{p}<0.05)$ and more dispersed $(18 / 20$, WMW test, $\mathrm{p}<0.05)$ movements in the big target compared

334 to the small target condition (figure 4A). Second, all subjects reached long targets with

335 significantly faster and longer movements compared to movements executed toward short targets

336 (WMW test, $\mathrm{p}<0.05$, figure 4B, left and middle panels). In this distance contrast, we observed

337 that endpoint distances from target center were not as consistently modulated as in the size

338 contrast, being significantly larger for the long target compared to the short target condition in

339 only 9 out of 20 subjects (WMW test, $\mathrm{p}<0.05$, figure $4 \mathrm{~B}$, right panel). The same influence of

340 target characteristics on reaching velocity, duration, and accuracy was found in the DR task (not

341 shown). Finally, the influence of target characteristics on movement parameters was similar in

342 the two experimental sessions and the two decision blocks (slow and fast, not shown).

343 To summarize, manipulating the target characteristics in distinct blocks of trials successfully

344 modulated reaching movement properties, encouraging subjects to either emphasize speed or

345 accuracy to execute movements in these blocks to express their choices. In the following section,

346 we assess whether or not these context-dependent adjustments of motor parameters influenced

347 the decision policy leading to the actions executed to report choices.

348 Effect of motor context on subjects' decision behavior

349 To determine the potential impact of movement context on decision policy, we first analyzed 350 subjects' decision duration (regardless of the decision outcome) by sorting trials depending on

351 target characteristics, irrespective of the session and the decision condition (slow or fast). 
Impact of a demanding movement on decision-making

352 By first comparing decisions made in big (big/short and big/long) versus small (small/short and

353 small/long) target trials, we found that decisions were overall shorter in the small target compared

354 to the big target condition (1099 versus $1154 \mathrm{~ms}$ ). Importantly, the difference is significant for

355 half of the population (WMW test, $\mathrm{p}<0.05$, figure $5 \mathrm{~A}$, left panel). Only one subject behaved the

356 opposite way, making significantly faster choices when allowed to report them with fast, less

357 accurate reaching movements. Importantly, we found virtually no difference between the average

358 decision difficulties (quantified as success probability profiles, see Methods and figure 2D) in the

359 two motor conditions, excluding a role of the sensory evidence experienced by the subjects in the

360 difference of decision duration observed between small and big target contexts. Did this

361 shortening of decision duration affect choice accuracy? To answer that question, we analyzed the

362 amount of sensory evidence that subjects needed to commit to their choices (i.e. their accuracy

363 criterion, computed as the sum of the log-likelihood ratios, see Methods), as a function of

364 decision duration for the two motor conditions, small and big target trials (Figure 5A, middle

365 panel). First, the level of sensory evidence that subjects required before committing to a choice

366 decreased as a function of decision duration, irrespective of motor conditions (ANCOVA,

367 SumLogLR, time effect, $\left.\mathrm{F}_{(1,347)}=164, \mathrm{p}<0.0001\right)$. This observation suggests that the more time

368 is elapsing over the time course of a trial, the more decisions rely on a sensory-agnostic signal. In

369 our previous studies as well as in others, this decreasing accuracy criterion is interpreted as a

370 behavioral signature of an urgency-gating mechanism of decision-making, which in short

371 describes the decision variable as the combination of sensory evidence with an urgency signal

372 and the decision is made when the decision variable reaches a constant threshold (Cisek et al.,

373 2009; Thura et al., 2012).

374 Importantly for the present report, we found that the accuracy criterion of subjects performing the

375 tokens task in small target trials was significantly lower than in big target trials, for any decision 
Impact of a demanding movement on decision-making

376 made after token jump \#3 (ANCOVA, SumLogLR, target size effect, $\mathrm{F}_{(1,347)}=4.63, \mathrm{p}=0.03$ ).

377 This indicates that subjects were more willing to tolerate less sensory evidence to make their

378 choices in small target compared to big target trials. As a consequence, decisions were usually

379 less likely to be correct in the small target compared to the big target context (Figure 5A, right

380 panel). This decrease of success probability in small target trials was significant in 7 out of 10

381 subjects showing a significant decrease of decision duration as a function of target size (WMW

382 test, $\mathrm{p}<0.05)$.

383 We next compared decision durations in short versus long target trials, a contrast that strongly

384 modulates movement speed of all subjects (Figure 4B, left panel). We found that the impact of

385 target distance, and thus movement speed, on decision duration was less consistent at the

386 population level compared to the impact of target size described above (Figure 5B, left panel).

387 Indeed, we observed that 6 subjects made significantly longer decisions in the short target

388 compared to the long target condition (WMW test, $\mathrm{p}<0.05$ ), 4 subjects behaved the opposite way

389 (WMW test, $\mathrm{p}<0.05$ ), and the 10 remaining ones did no behave differently, in terms of decision

390 duration, between the two motor conditions. We also found that target distance did not

391 significantly influence the quantity of sensory information used by subjects to commit to their

392 choice (ANCOVA, SumLogLR, target size effect, $\mathrm{F}_{(1,346)}=0.13, \mathrm{p}=0.72$, Figure 5B, middle

393 panel), and the success probability of these choices was only rarely significantly modulated as a

394 function of target distance (Figure 5B, right panel).

395 We next analyzed the effect of target size and distance on subjects' reaction times (RT) in the

396 delayed reach (DR) task. In the DR task, no volitional commitment needed to be made as subjects

397 were instructed with both the correct target and when to execute their response (see Methods). In

398 this task, we found that subjects' RTs were overall longer in small target compared to big target 
Impact of a demanding movement on decision-making

399 trials (375 versus $367 \mathrm{~ms}$ ), with a significant difference for 8 out of 20 subjects (WMW test, $400 \mathrm{p}<0.05$ ), and only one subject behaving significantly the opposite way (Figure 5C). Interestingly,

401 we found a significant correlation between the modulation of decision duration by target size in

402 the tokens task and the modulation of reaction time in the same conditions in the DR task. In 403 other words, the more subjects expedited decisions in the small target condition of the tokens

404 tasks, the more they slowed down their response initiation in the same condition in the DR task

405 (Pearson correlation, $\mathrm{r}=-0.495, \mathrm{p}=0.026$, Figure 6). By contrast, reaction times were less

406 homogeneously affected by the distance condition in the DR task. Four subjects reacted faster in

407 short compared to long target blocks, and 3 subjects behaved the opposed way (WMW test, $408 \mathrm{p}<0.05$, Figure 5D).

409 To assess whether the effect of target size on decision policy was dependent on the decision 410 context, i.e. the slow or fast decision blocks of the tokens task, we computed subjects' decision 411 duration, success probability, and sensory evidence at decision time for each of the two size 412 conditions, separately for the two decision blocks. In a recent report (Thura, 2020), we describe 413 in detail subjects' behavior in the two decision conditions. Quickly, the "slow" decision block of 414 trials encourages slow and accurate decisions because the tokens that remain in the central 415 decision circle after movement completion accelerate only a little compared to the pre-decision 416 period (see Methods).

417 By contrast, in the "fast" block of trials, the remaining tokens accelerate a lot, allowing subjects 418 to potentially save a lot of time by deciding quickly, permitting to eventually maximize their 419 reward rate. In Thura, 2020 we showed that subjects behaved accordingly, making faster (1028 vs $4201229 \mathrm{~ms}$ across subjects) and less accurate (0.87 versus 0.97$)$ decisions in the fast block compared 421 to the slow block of trials (see the average distributions of decision duration across subjects in the 
Impact of a demanding movement on decision-making

422 two decision blocks in Figure 7A,B). In the present report, we demonstrate that the impact of

423 target size on decision policy, especially accuracy, is larger in the slow block than in the fast

424 block of trials. Indeed, decision durations were significantly modulated by target size in 8 out of

42520 subjects performing the slow block whereas they were modulated in only 6 subjects

426 performing the tokens task in the fast condition (WMW test, $\mathrm{p}<0.05$ ). Moreover, the accuracy

427 criterion was significantly higher for big target compared to small target trials in the slow block

428 (ANCOVA, SumLogLR, size effect, $\left.\mathrm{F}_{(1,345)}=13.6, \mathrm{p}=0.0003\right)$ but not in the fast block $\left(\mathrm{F}_{(1,298)}=\right.$

$4290.1, \mathrm{p}=0.75$, Figure 7A,B, left panels). As a consequence, success probability was strongly

430 influenced by target size in the slow block (significantly modulated in 9 out of 20 subjects,

431 WMW test, $\mathrm{p}<0.05$ ) whereas effects were more balanced in the fast blocks (Figure 7A,B, right

432 panels).

433 Next, we analyzed the effect of target size on decision policy depending on the level of

434 experience of subjects in the tokens task. To do this, we computed subjects' decision duration,

435 success probability, and sensory evidence at decision time for decisions made in the slow

436 decision block for each of the two target size conditions, separately for the two experimental

437 sessions. Overall, we found that the impact of the target size on decision policy did not strongly

438 evolve with training. Decision durations were slightly more modulated by target size in the first

439 session than in the second sessions (5/20 and 3/20 subjects with a significant effect of target size

440 on decision duration in session $\# 1$ and $\# 2$, respectively; WMW test, $\mathrm{p}<0.05$ ), but accuracy

441 criterion (ANCOVA, SumLogLR, size effect, $F_{(1,320)}=2.5, p=0.1$ in session $\# 1 ; F_{(1,330)}=10.5$,

$442 \mathrm{p}=0.0013$ in session \#2) and to a lesser extent, success probability (4/20 and 5/20 subjects with a

443 significant effect of target size on decision duration in session \#1 and \#2, respectively; WMW 
Impact of a demanding movement on decision-making

444 test, $\mathrm{p}<0.05$ ) were more affected by target size in session \#2 compared to session \#1 (Figure $4457 \mathrm{C}, \mathrm{D})$.

446 Finally, we evaluated the impact of the faster and less accurate choices in the small target

447 condition compared to the big target condition on subjects' performance in the tokens task.

448 Because it has been shown that subjects seek to optimize their rate of correct responses rather

449 than their absolute accuracy (Balci et al., 2011), performance is estimated as the duration that 450 subjects needed to complete each motor block. Thus, by calculating the rate of reward and 451 deducting from it the amount of time necessary to complete the different motor blocks in each

452 session (see Methods), we found that this duration was significantly longer in the small target 453 condition compared to the big target condition across subjects, regardless of the session 454 performed, when subjects performed the tokens task in the slow decision block (WMW test, $\mathrm{p}=$ 455 0.0013, Figure 8, left panel). By contrast, we found no significant difference in block duration 456 between small and big target conditions in the fast decision block of trials (WMW test, $\mathrm{p}=0.11$, 457 Figure 8, right panel).

\section{DISCUSSION}

460 In this study, we assessed whether the motor context in which perceptual decisions between 461 actions are made influences human subjects' decision strategy, as predicted by the recently 462 proposed "shared regulation" hypothesis (Thura et al., 2014). This model conceives decision and 463 action as a continuum, regulated by unspecific signals. As a consequence, a motor context 464 favoring vigorous movements should be preceded by fast decisions because of the activation of 465 one unique invigoration signal possibly computed in the basal ganglia (Cisek and Thura, 2018).

466 We found that motor context indeed often influences decision-making but contrary to the 
Impact of a demanding movement on decision-making

467 prediction of the shared regulation hypothesis, decisions preceding slow and accurate actions

468 were faster, rather than slower, compared to decisions made in blocks allowing more vigorous

469 and less accurate actions.

470 In the present task, the action vigor (indicated by movement speed and duration) is assumed to be

471 determined depending on the speed-accuracy trade-off of each motor condition. However,

472 motivation factors, such as the movement energetic cost, may have contributed to shape action

473 vigor as well (Mazzoni et al., 2007). With the present design, we cannot disentangle the

474 contribution of the accuracy and energy costs on vigor definition, the slower movements

475 executed toward the small targets being also less energetically costly. However, if we assume that

476 the less energetically costly movements should increase the subjects' implicit motivation to

477 decide and act (Mazzoni et al., 2007), those movements should be executed faster than the

478 effortful ones. Yet, our data indicate the opposite results. We thus believe that the accuracy

479 requirement is the main factor that determined movement vigor in our experiment.

480 Motor costs influence motor and perceptual decision-making

481 The present results first add to the many recent observations that challenge the classic view of

482 behavior organization, inherited from cognitive psychology, in which perception, decision, and

483 action are considered as temporally separate and serial processes (Pylyshyn, 1984). Indeed, in

484 ecological scenarios, sensory or value-based decisions are very often expressed by actions that

485 are themselves associated with risks and costs. For instance, a monkey deciding between reaching

486 toward a grape or a nut may prefer the nut but time and energy expenditure associated with

487 opening its shell may rather encourage him to go for the grape. Because it has been extensively

488 demonstrated that the brain tends to control behavior in such a way that the expected value of a

489 choice is maximized while all types of cost are minimized (Neumann and Morgenstern, 1944; 
Impact of a demanding movement on decision-making

490 Todorov and Jordan, 2002; Gold and Shadlen, 2007; Christopoulos and Schrater, 2015;

491 Christopoulos et al., 2015; Diamond et al., 2017), any potentially penalizing factor, including

492 motor costs, should influence the perceptual judgment leading to a potential reward.

493 In the past decade, several studies have demonstrated that motor costs influence decision-making

494 when choices only rely on movement properties (i.e. motor decisions). Cos and colleagues

495 showed that when humans make rapid choices between reaching actions, they tend to choose the

496 one that carries the lowest biomechanical cost (Cos et al., 2011, 2014). Morel and colleagues

497 found that biomechanics affects action selection too, but among duration, amplitude, direction

498 and force, they observed that movement duration is perceived as the greatest cost by subjects

499 (Morel et al., 2017). Finally, Michalski and colleagues observed that movement amplitude and

500 direction influence the probability of switching from one ongoing movement to another in a

501 common real-life scenario where one has to decide while already acting (Michalski et al., 2020).

502 Other work addressed the effects of motor costs on decision-making beyond purely motor

503 choices, i.e. when the decision primarily relies on perceptual or value information, as in the

504 present work. In three of these experiments using the random dots motion discrimination task,

505 data indicate an effect of motor constraints on non-motor decision-making. Burk and colleagues

506 demonstrated that physical effort affects the proportion of changes of mind made by subjects

507 during the deliberation period: the more the change of mind requires a significant energetic cost,

508 the less subjects are willing to perform it (Burk et al., 2014). Another study showed that

509 asymmetric biomechanical cost biases perceptual decisions, with subjects more systematically

510 choosing targets associated with movements of lower cost, even if these choices were detrimental

511 to accuracy (Marcos et al., 2015). In agreement with this observation, Hagura and colleagues

512 demonstrated that motion discrimination is influenced by the physical resistance applied to the 
Impact of a demanding movement on decision-making

513 response. Intriguingly, they showed that motor costs also bias vocally-expressed judgments,

514 suggesting that actions changed how subjects perceived the stimuli themselves (Hagura et al.,

515 2017). It is important to note that in these three studies, each of the two potential targets was

516 assigned a specific motor cost during a given choice. By contrast, in the present work, the two

517 targets were always associated with the same motor cost, and that cost was varied between blocks

518 of trials. The present report is thus to our knowledge the first to show that the motor context in

519 which a movement is performed influences the strategy of subjects during decision-making.

520 A flexible mechanism for regulating decision and movement durations

521 Decisions about actions typically include a period of deliberation that ends with the commitment

522 to a choice, which then leads to the overt expression of that choice through action execution, at

523 the end of which the reward can be at last consumed. Because decision and action processes are

524 so inextricably linked, it is natural to imagine that they could at least partly share operating

525 principles to maximize the utility of behavior. Decision and action could indeed be considered as

526 a continuum during which regulation signals would affect both processes agnostically, in a

527 unified manner. In agreement with this hypothesis, it has been proposed that movement selection,

528 preparation, and execution are parameterized following economical rules, varying depending on

529 utility estimation: high valued options lead to faster reaction times and movement speed, and

530 high-perceived effort discount option's value, leading to slower reaction and longer movements

531 (Kawagoe et al., 1998; Wickler et al., 2000; Shadmehr et al., 2010, 2016, 2019; Haith et al.,

532 2012; Choi et al., 2014; Morel et al., 2017; Reppert et al., 2018; Summerside et al., 2018; Yoon et

533 al., 2018; Revol et al., 2019).

534 Our previous results support this hypothesis of a coordination between decision and action

535 durations during behavior. For instance, within fixed decision and motor contexts, both humans 
Impact of a demanding movement on decision-making

536 and monkeys shorten their movement duration in trials in which decision duration is prolonged,

537 as if extended deliberation duration was compensated by increasing the action speed so that the

538 next opportunity can be encountered more quickly. Between decision contexts, choices made in a

539 fast speed-accuracy trade-off regime are usually followed by faster movements compared to

540 those made in a regime encouraging slow and accurate choices (Thura et al., 2014; Thura, 2020).

541 Altogether, these observations indicate that the level of urgency at which a decision is made

542 directly influences movement vigor, suggesting that decision and movement durations are

543 determined by a global decision urgency/movement vigor signal that invigorates behavior in

544 order to control reward rate (Cisek and Thura, 2018; Carland et al., 2019). However, a missing

545 test of the shared regulation hypothesis required to vary the motor context in which a decision is

546 made and assess whether or not a motor context permitting execution of vigorous movements to

547 express choices leads to faster decisions compared to the same difficult decisions made in a

548 demanding motor context, imposing slow and accurate movements. Contrary to this prediction,

549 we did not observe a robust and consistent effect of movement speed per se on decision duration

550 and accuracy (by comparing short versus long target conditions, Figure 5B). Instead, data

551 indicate that target size imposes a motor accuracy cost that is tackled by some subjects by

552 shortening the deliberation period (Figure 5A) so that more time is available to prepare the

553 following movement execution. This interpretation is supported by a post-experiment interview

554 during which most of the participants declared having consciously expedited and thus

555 "sacrificed" their decisions to better prepare action execution in small target trials.

556 One critical assumption in this experiment is that action towards the smaller targets requires less

557 vigor compared to the action executed toward large targets. However, an alternative

558 interpretation would state that because small targets impose more preparation time (reaction 
Impact of a demanding movement on decision-making

559 times are overall longer for small targets than for big targets in the DR task, figure 5C), a

560 potential preparation-related urgency would have more time to increase in the small target blocks

561 compared to the big target blocks, leading to movements initiated under higher urgency in the

562 small blocks compare to the big blocks. The slower velocity and longer movement duration

563 observed in the small blocks (Figure 4A for the tokens task) would then be explained by possible

564 different systems for governing action preparation and execution (e.g. Haith et al., 2016).

565 However, we do not believe that a putative preparation-related urgency signal could explain our

566 results because no influence of this urgency is expected at the beginning of the trial and during

567 the deliberation process. Instead, the preparation-related urgency level might differ between the

568 blocks only after commitment, i.e. during movement preparation.

569 Thus, the present results more likely demonstrate that an unconditional and unidirectional

570 relationship between action vigor and decision duration, as predicted by the shared regulation

571 hypothesis, is absent. Instead, our results claim for a flexible mechanism in which decision and

572 action durations are regulated by independent, yet interacting, decision urgency and movement

573 vigor signals. Such flexibility is certainly advantageous given the inherent complexity of the

574 many variables interrelationships at play during goal-directed behavior, where no single decision

575 policy is guaranteed to maximize the reward rate across all contexts.

576 Flexibility between decision-making and action execution is well illustrated by the relationship

577 between the effect of target size on decision duration in the tokens task and the effect of target

578 size on reaction time in the delayed reach (DR) task. The significant correlation (Figure 6)

579 indicates that subjects who are slower to initiate a movement in the small target trials of the DR

580 task are also the subjects who adjust their decision policy the most in these difficult trials in the

581 tokens task. The former result is consistent with data suggesting that effortful movements 
Impact of a demanding movement on decision-making

582 discount reward value, thus motivation, delaying the initiation of movements (Mazzoni et al.,

583 2007; Summerside et al., 2018; Shadmehr et al., 2019). This relationship thus suggests that

584 economic principles governing behavior utility in non-decision tasks extend to decision-making.

585 It also indicates that when the task difficulty mainly relies on movement execution, as in the DR

586 task, movement effort slows down reaction times whereas when task difficulty is shared between

587 decision and action, as in the tokens task, movement effort influences the decision process in an

588 opposite way. What could be the relevance of this intriguing behavior in terms of performance?

Impact of a demanding movement on reward rate

590 The present data indicate that movement accuracy requirements, more than speed or duration,

591 forced some subjects to hasten their decisions. It seems that they took advantage of the

592 potentially long deliberation period permitted in the task (up to $3 \mathrm{~s}$ ) to sometimes shorten their

593 judgment in order to focus on the following movement execution. Interestingly, such adjustment

594 only occurred in blocks of trials in which decisions were encouraged to be conservative ("slow"

595 decision blocks, Figure 7). Indeed, the large and very profitable, in terms of reward rate,

596 shortening of decision durations observed in the "fast" decision blocks (Figure 8) probably

597 constrained decision policy too much, preventing any other adjustments of behavior. It is also

598 important to remember that in the tokens task, deciding more quickly does not provide additional

599 time to execute the movement, the maximum movement duration being fixed at $800 \mathrm{~ms}$ regardless

600 of subjects' reach onset timing. How then can one explain this suboptimal strategy? One

601 possibility is that our limited cognitive and motor resources imposed a necessary trade-off

602 between decision and action when task constraints were too demanding (Wickens, 2002). In this

603 view, subjects had to choose between allocating resources on decision-making while taking the

604 risk of producing inaccurate movements or rather sacrificing decision-making to presumably 
Impact of a demanding movement on decision-making

605 better prepare and execute their movements. Knowing that in ecological situations as in the

606 present task, a movement usually follows the decision, it is possible that subjects gave priority to

607 the action process considering that movement failure would prevent reward acquisition even if

608 the decision was correct. Although it may be advantageous in terms of reward rate to decide very

609 quickly while sacrificing a little bit of precision (see equation 3), as observed when humans and

610 monkeys decide faster in the fast compared to the slow decision block of trials (Figure 8 and

611 Thura et al., 2014; Thura, 2020), our results show however that the strategy consisting of

612 sacrificing decision accuracy to execute accurate movements led to a drop of reward rate

613 compared to a condition in which such adjustment was not necessary. This is probably because in

614 small target trials, the probability of choosing the correct target decreased, even if the amount of

615 time saved during the deliberation period compensated the longer movements made in this

616 condition (Figure 4).

617 Possible neurophysiological origin of the decision and action regulation mechanism

618 The interaction between the decision and action regulations provides a clue to the neural origins

619 of the signals implicated in this mechanism. Interacting decision urgency and movement vigor

620 signals would be expected to originate from a region that projects to a wide range of cortical

621 areas to influence both decision-making and action execution. In this respect, the basal ganglia

622 (BG) provide a natural candidate. The BG have long been functionally associated with the

623 regulation of motivated behavior and reinforcement learning for maximizing reward (Graybiel,

624 2005; Frank, 2011), and multiple lines of neuropsychological, neurological and

625 neurophysiological evidence suggest that effort expenditure and movement vigor are largely

626 under the control of activity within a variety of BG structures, including the striatum, substantia

627 nigra, ventral pallidum, and the globus pallidus (Mazzoni et al., 2007; Turner and Desmurget, 
Impact of a demanding movement on decision-making

628 2010; Rueda-Orozco and Robbe, 2015; Dudman and Krakauer, 2016; Thura and Cisek, 2017; da

629 Silva et al., 2018; Yttri and Dudman, 2018; Carland et al., 2019; Fobbs et al., 2020). All these

630 studies along with results from the present report suggest a mechanism in which different

631 populations of cells, located in the BG output nuclei, vary their activity to adjust both decision

632 and motor durations under specific circumstances, in order to control the rate of reward. Future

633 experiments designed to record the activity of individual BG cells during decision-making

634 between actions in different decision and motor contexts should allow us to better understand the

635 neural correlates of this regulation mechanism.

\section{$636 \quad$ Limitations}

637 A limitation of the present study, as often in investigations of primate cognition and behavior, 638 relates to the between-subject variability of the results. The average decision duration ranges 639 from $\sim 700 \mathrm{~ms}$ to about $1600 \mathrm{~ms}$ depending on subjects (Figure 5), even though participants faced 640 the same trials under identical conditions. This indicates individual "traits" of decision behavior.

641 Similarly, a subgroup of four subjects was more vigorous than the others to execute their 642 movements (Figure 4). While revealing probable unaddressed phenomena, these multiple levels 643 of variability are still compatible with a flexible regulation mechanism of decision and action 644 durations that would be idiosyncratic in nature. Another limitation concerns the absence of 645 analysis of decision data in inaccurate or slow movement trials for methodology reasons. In the 646 present report, we show that a difficult movement is often preceded by a fast and inaccurate 647 decision, but this occurs when movements are properly executed. It is possible that subjects 648 sometimes allocated their attention on the decision process, leading in that case to a "sacrifice" of 649 motor control, resulting in failed movements. Further experiments or analyses are needed to 
bioRxiv preprint doi: https://doi.org/10.1101/2020.04.08.028936; this version posted July 7, 2020. The copyright holder for this preprint (which

was not certified by peer review) is the author/funder, who has granted bioRxiv a license to display the preprint in perpetuity. It is made available under ACC-BY-NC-ND 4.0 International license.

Impact of a demanding movement on decision-making

650 reveal which of the two processes, the decision or the action, is typically prioritized by

651 participants in this kind of demanding goal-directed behavior.

652

653

\section{ACKNOWLEDGMENTS}

654 The authors wish to thank Sonia Alouche and Jean-Louis Borach for effective administrative

655 assistance, Paul Cisek for his contribution in setting up the software environment, Frédéric

656 Volland for his expertise during the technical preparation of this experiment, and Martine

657 Meunier for helpful suggestions on the manuscript. 
Impact of a demanding movement on decision-making

\section{FIGURE CAPTIONS}

660 Figure 1: The "shared regulation" hypothesis. A. Simplified hypothetical mechanism of a

661 shared regulation of decision and movement durations by one unique invigoration (decision

662 urgency/movement vigor) signal, possibly computed in the basal ganglia (Thura et al., 2014;

663 Thura and Cisek, 2017). The thick black lines illustrate the manipulation of the motor context,

664 tested in the present study, leading to the modulation of the urgency/vigor signal. B. The shared

665 regulation hypothesis makes a simple prediction regarding the effect of the motor context in

666 which a decision is made on the duration of that decision: if a context encourages execution of

667 vigorous (faster, shorter) movements (orange) to report choices, then the urgency level in this

668 context should be raised compared to another context in which movements need to be less

669 vigorous but more accurate (blue). As a consequence, equally difficult decisions made in the

670 vigorous block of trials should be on average shorter than those made in the block encouraging

671 slow and accurate movements.

672

673 Figure 2: Apparatus, experimental design, and conditions. A. Experimental apparatus. B.

674 Time course of a trial in the tokens task. C. Motor conditions, i.e. movement target size and 675 distance combinations. In distinct blocks of trials, both lateral targets could be either small and 676 located close to the starting circle (black), small and located far from the starting circle, big and 677 located close to the starting circle or big and located far from the starting circle. D. Average 678 success probability profiles of trials experienced by subjects in each of the four motor conditions.

680 Figure 3: Motor behavior in one example subject. A. Panel shows the motor visual display 681 depicted in Figure 2, along with shaded ellipses illustrating for each motor condition and side 
Impact of a demanding movement on decision-making

682 with respect to the start circle (black) the dispersion (an iso-contour of the Gaussian distribution)

683 of one example subject reaching endpoints in the tokens task. Each ellipse contains $95 \%$ of the

684 data in each condition, and trials include correct and inadequate (too slow or inaccurate)

685 movements executed in the two sessions and the two decision conditions (slow and fast). B.

686 Reach velocity profiles of the same subject in the four motor conditions. Same color/style

687 convention as in A. Only adequate movements are included.

689 Figure 4: Effect of motor context on population motor behavior. A. Average reaching

690 movement peak velocity (left), duration (middle) and target center-endpoint distance (right) of

691 each subject during big target (big/short and big/long blocks, x-axis) and small target (small/short

692 and small/long blocks, y-axis) conditions performed in the tokens task. Green (magenta) pluses

693 indicate the mean and SE for subjects for whom data is larger (smaller) in the big target condition

694 compared to the small target condition and the difference was significant (WMW test, $\mathrm{p}<0.05$ ).

695 Data include trials collected from both sessions \#1 and \#2, in both the slow and fast decision

696 blocks. B. Same as A for trials executed in the long target (small/long and big/long blocks, x-

697 axis) versus the short target (small/short and big/short blocks, y-axis) condition.

699 Figure 5 : Effect of motor context on decision behavior. A. Left: Average decision duration of

700 each subject during big (x-axis) and small (y-axis) target conditions performed in the tokens task.

701 Same convention as in Figure 4. Middle: Average ( \pm SE) evidence at decision time across

702 subjects as a function of decision duration in the small (blue) and the big (orange) target

703 conditions of the tokens task. Right: Mean success probability of each subject during big (x-axis)

704 and small (y-axis) target conditions performed in the tokens task. Same convention as in Figure 4. 
Impact of a demanding movement on decision-making

705 Data include trials collected from both sessions \#1 and \#2, in both the slow and fast decision

706 blocks. B. Same as A for trials executed in the long versus short target conditions. C. Average

707 reaction time of each subject during big (x-axis) and small (y-axis) target conditions performed in

708 the delayed reach task. D. Same as C for trials executed in the long (x-axis) versus the short (y-

709 axis) target condition.

711 Figure 6: Relationship between the effect of motor context on the decision and instructed

712 tasks. Left: Correlation between the difference of decision duration in small versus big target

713 conditions in the tokens task (x-axis) and the difference of reaction time in the same conditions in

714 the delayed reach task (y-axis). Each dot shows data from one individual subject. Right: Same as

715 Left for the distance contrast (short versus long target conditions).

717 Figure 7: Effect of motor context on decision accuracy depending on decision context and

718 experience. A. Left: Average $( \pm \mathrm{SE})$ evidence at decision time across subjects as a function of

719 decision duration in small (blue) and big (orange) target conditions performed in the "slow"

720 decision block of the tokens task. The black line below shows the average distribution of decision

721 duration across subjects in the slow block. Right: Average success probability of each subject

722 during big (x-axis) and small (y-axis) target conditions performed in the slow decision block of

723 the tokens task. Data from both sessions \#1 and \#2 are included. Same convention as in Figure 4.

724 B. Same as A for decisions made in the "fast" decision block of the tokens task. C. Same as A for

725 decisions made in the first session, including only slow decision blocks. D. Same as C for

726 decisions made during the second session. 
bioRxiv preprint doi: https://doi.org/10.1101/2020.04.08.028936; this version posted July 7, 2020. The copyright holder for this preprint (which

was not certified by peer review) is the author/funder, who has granted bioRxiv a license to display the preprint in perpetuity. It is made available under aCC-BY-NC-ND 4.0 International license.

Impact of a demanding movement on decision-making

728 Figure 8: Influence of target size on the expected duration of blocks. Bars show the average

729 expected time necessary to complete a block of 80 trials, computed based on reward rate in each

730 condition, in the small (blue) and big (orange) target block across subjects and sessions, in the

731 slow (left) and fast (right) decision block of trials. Dots illustrate individual data.

732 
Impact of a demanding movement on decision-making

\section{REFERENCES}

734 Balci F, Simen P, Niyogi R, Saxe A, Hughes JA, Holmes P, Cohen JD (2011) Acquisition of decision making criteria: reward rate ultimately beats accuracy. Atten Percept Psychophys 73:640-657.

Bogacz R, Hu PT, Holmes PJ, Cohen JD (2010) Do humans produce the speed-accuracy tradeoff that maximizes reward rate? Quarterly Journal of Experimental Psychology 63:863891. Changes of Mind in Sensorimotor Decision Making Kiebel S, ed. PLoS ONE 9:e92681.

742 Carland MA, Thura D, Cisek P (2019) The Urge to Decide and Act: Implications for Brain Function and Dysfunction. Neuroscientist:107385841984155.

744 Choi JES, Vaswani PA, Shadmehr R (2014) Vigor of Movements and the Cost of Time in Decision Making. Journal of Neuroscience 34:1212-1223.

746 Christopoulos V, Bonaiuto J, Andersen RA (2015) A biologically plausible computational theory for value integration and action selection in decisions with competing alternatives. PLoS Comput Biol 11:e1004104. 11:e1004402. 
Impact of a demanding movement on decision-making

752 Churchland AK, Kiani R, Shadlen MN (2008) Decision-making with multiple alternatives. Nat $753 \quad$ Neurosci 11:693-702.

754 Cisek P, Puskas GA, El-Murr S (2009) Decisions in Changing Conditions: The Urgency-Gating $755 \quad$ Model. Journal of Neuroscience 29:11560-11571.

756 Cisek P, Thura D (2018) Neural circuits for action selection. In: Reach-to-grasp behavior: Brain, 757 behavior, and modelling across the life span, Daniela Corbetta and Marco Santello., pp 758 91-118 Frontiers of developmental science. Taylor \& Francis Group.

759 Cos I, Bélanger N, Cisek P (2011) The influence of predicted arm biomechanics on decision making. Journal of Neurophysiology 105:3022-3033.

761 Cos I, Duque J, Cisek P (2014) Rapid prediction of biomechanical costs during action decisions. $762 \quad$ Journal of Neurophysiology 112:1256-1266.

763 Cos I, Medleg F, Cisek P (2012) The modulatory influence of end-point controllability on 764 decisions between actions. Journal of Neurophysiology 108:1764-1780.

765 da Silva JA, Tecuapetla F, Paixão V, Costa RM (2018) Dopamine neuron activity before action 766 initiation gates and invigorates future movements. Nature 554:244-248.

767 Diamond JS, Wolpert DM, Flanagan JR (2017) Rapid target foraging with reach or gaze: The 768 hand looks further ahead than the eye. PLoS Comput Biol 13:e1005504.

769 Ditterich J (2006) Evidence for time-variant decision making. European Journal of Neuroscience 770 $24: 3628-3641$. 
Impact of a demanding movement on decision-making

771 Dudman JT, Krakauer JW (2016) The basal ganglia: from motor commands to the control of vigor. Current Opinion in Neurobiology 37:158-166.

773 Fobbs WC, Bariselli S, Licholai JA, Miyazaki NL, Matikainen-Ankney BA, Creed MC, Kravitz Neurosci 40:1679-1688.

776 Frank MJ (2011) Computational models of motivated action selection in corticostriatal circuits. Current Opinion in Neurobiology 21:381-386. 574. Neurobiology 15:638-644.

Hagura N, Haggard P, Diedrichsen J (2017) Perceptual decisions are biased by the cost to act. eLife 6:e18422. Movement Initiation. J Neurosci 36:3007-3015. Reward in Control of Movements. Journal of Neuroscience 32:11727-11736.

788 Kawagoe R, Takikawa Y, Hikosaka O (1998) Expectation of reward modulates cognitive signals in the basal ganglia. Nat Neurosci 1:411-416. 
Impact of a demanding movement on decision-making

Kira S, Yang T, Shadlen MN (2015) A Neural Implementation of Wald's Sequential Probability Ratio Test. Neuron 85:861-873. decision boundary. Journal of Experimental Psychology: General 146:776-805.

794 Malhotra G, Leslie DS, Ludwig CJH, Bogacz R (2018) Time-varying decision boundaries: 795 insights from optimality analysis. Psychon Bull Rev 25:971-996.

796 Marcos E, Cos I, Girard B, Verschure PFMJ (2015) Motor Cost Influences Perceptual Decisions 797 Gribble PL, ed. PLoS ONE 10:e0144841.

798 Mazzoni P, Hristova A, Krakauer JW (2007) Why Don’t We Move Faster? Parkinson’s Disease, 799 Movement Vigor, and Implicit Motivation. Journal of Neuroscience 27:7105-7116.

800 Michalski J, Green AM, Cisek P (2020) Reaching decisions during ongoing movements. Journal 801 of Neurophysiology 123:1090-1102.

802 Morel P, Ulbrich P, Gail A (2017) What makes a reach movement effortful? Physical effort 803 discounting supports common minimization principles in decision making and motor $804 \quad$ control Rushworth M, ed. PLoS Biol 15:e2001323.

805 Murphy PR, Boonstra E, Nieuwenhuis S (2016) Global gain modulation generates time806 dependent urgency during perceptual choice in humans. Nat Commun 7:13526.

807 Myerson J, Green L (1995) Discounting of delayed rewards: models of individual choice. Journal 808 of the Experimental Analysis of Behavior:263-276. 
Impact of a demanding movement on decision-making

809 Neumann J von, Morgenstern O (1944) Theory of Games and Economic Behavior, Princeton $810 \quad$ University Press.

811 Reppert TR, Rigas I, Herzfeld DJ, Sedaghat-Nejad E, Komogortsev O, Shadmehr R (2018) 812 Movement vigor as a traitlike attribute of individuality. Journal of Neurophysiology $813 \quad 120: 741-757$.

814 Revol P, Collette S, Boulot Z, Foncelle A, Niki C, Thura D, Imai A, Jacquin-Courtois S, Cabanac 815 M, Osiurak F, Rossetti Y (2019) Thirst for Intention? Grasping a Glass Is a Thirst$816 \quad$ Controlled Action. Front Psychol 10:1248.

817 Rueda-Orozco PE, Robbe D (2015) The striatum multiplexes contextual and kinematic 818 information to constrain motor habits execution. Nat Neurosci 18:453-460.

819 Shadmehr R, Huang HJ, Ahmed AA (2016) A Representation of Effort in Decision-Making and $820 \quad$ Motor Control. Current Biology 26:1929-1934.

821 Shadmehr R, Orban de Xivry JJ, Xu-Wilson M, Shih T-Y (2010) Temporal Discounting of 822 Reward and the Cost of Time in Motor Control. Journal of Neuroscience 30:10507$823 \quad 10516$.

824 Shadmehr R, Reppert TR, Summerside EM, Yoon T, Ahmed AA (2019) Movement Vigor as a 825 Reflection of Subjective Economic Utility. Trends in Neurosciences 42:323-336.

826 Standage D, You H, Wang D-H, Dorris MC (2011) Gain Modulation by an Urgency Signal 827 Controls the Speed-Accuracy Trade-Off in a Network Model of a Cortical Decision 828 Circuit. Front Comput Neurosci 5 Available at: 
Impact of a demanding movement on decision-making September 9, 2019].

831 Steinemann NA, O’Connell RG, Kelly SP (2018) Decisions are expedited through multiple neural adjustments spanning the sensorimotor hierarchy. Nat Commun 9:3627.

833 Summerside EM, Shadmehr R, Ahmed AA (2018) Vigor of reaching movements: reward discounts the cost of effort. Journal of Neurophysiology 119:2347-2357.

835 Thura D (2020) Decision urgency invigorates movement in humans. Behavioural Brain Research 836 $382: 112477$.

Thura D, Beauregard-Racine J, Fradet C-W, Cisek P (2012) Decision making by urgency gating: theory and experimental support. J Neurophysiol 108:2912-2930. during dynamic decision making. Neuron 81:1401-1416. Volitional Adjustments of Speed-Accuracy Trade-Offs. J Neurosci 36:938-956.

843 Thura D, Cisek P (2017) The Basal Ganglia Do Not Select Reach Targets but Control the Urgency of Commitment. Neuron 95:1160-1170.e5.

845 Thura D, Cos I, Trung J, Cisek P (2014) Context-dependent urgency influences speed-accuracy trade-offs in decision-making and movement execution. J Neurosci 34:16442-16454.

847 Todorov E, Jordan MI (2002) Optimal feedback control as a theory of motor coordination. Nat $848 \quad$ Neurosci 5:1226-1235. 
Impact of a demanding movement on decision-making

849 Turner RS, Desmurget M (2010) Basal ganglia contributions to motor control: a vigorous tutor.

$850 \quad$ Current Opinion in Neurobiology 20:704-716.

851 Wickens CD (2002) Multiple resources and performance prediction. Theoretical Issues in

$852 \quad$ Ergonomics Science 3:159-177.

853 Wickler SJ, Hoyt DF, Cogger EA, Hirschbein MH (2000) Preferred speed and cost of transport.

$854 \quad$ Journal of Experimental Biology:2195-2200.

855 Yoon T, Geary RB, Ahmed AA, Shadmehr R (2018) Control of movement vigor and decision making during foraging. Proc Natl Acad Sci USA 115:E10476-E10485.

857 Yttri EA, Dudman JT (2018) A Proposed Circuit Computation in Basal Ganglia: History-

858 Dependent Gain: Proposed Circuit Computation in Basal Ganglia. Mov Disord 33:704-

859716.


A

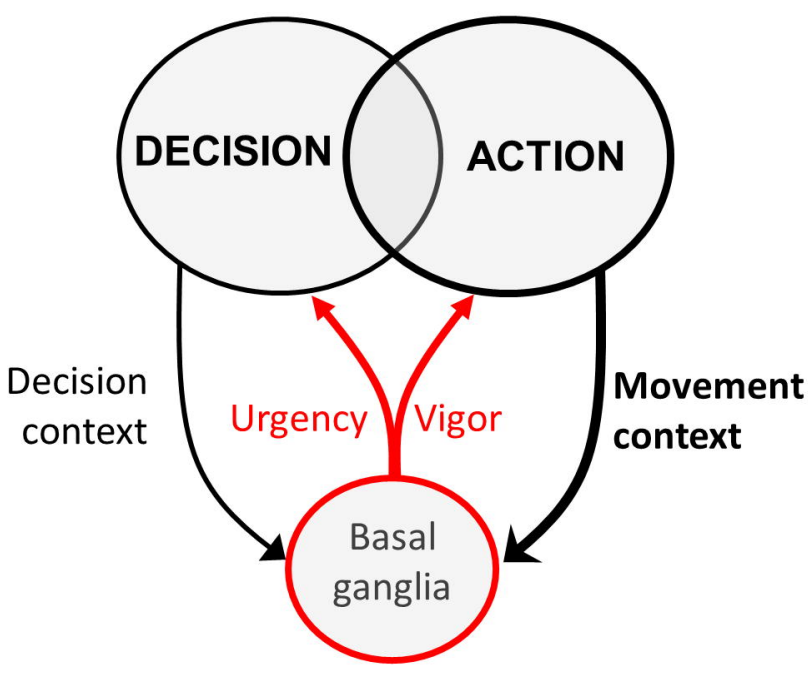

B

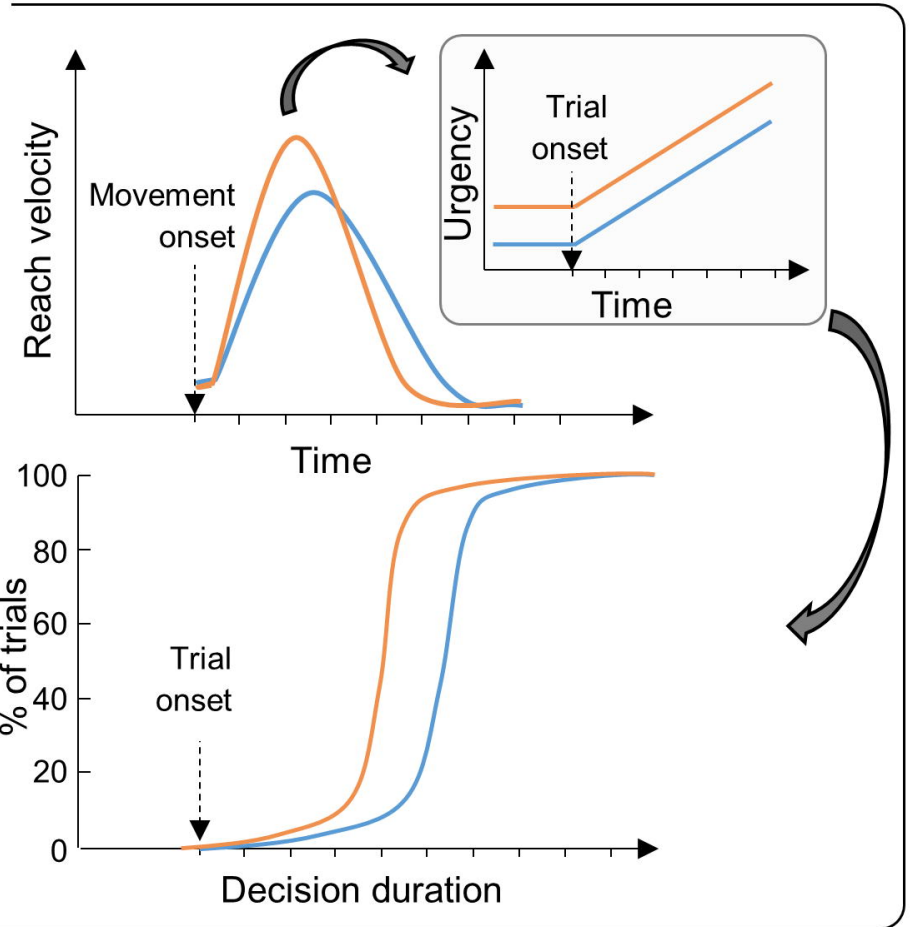


$\mathbf{A}-$

Subject $\mathbf{S 6}$

$$
\text { — Small-short block }
$$

Small-long block

_Big-short block

Big-long block
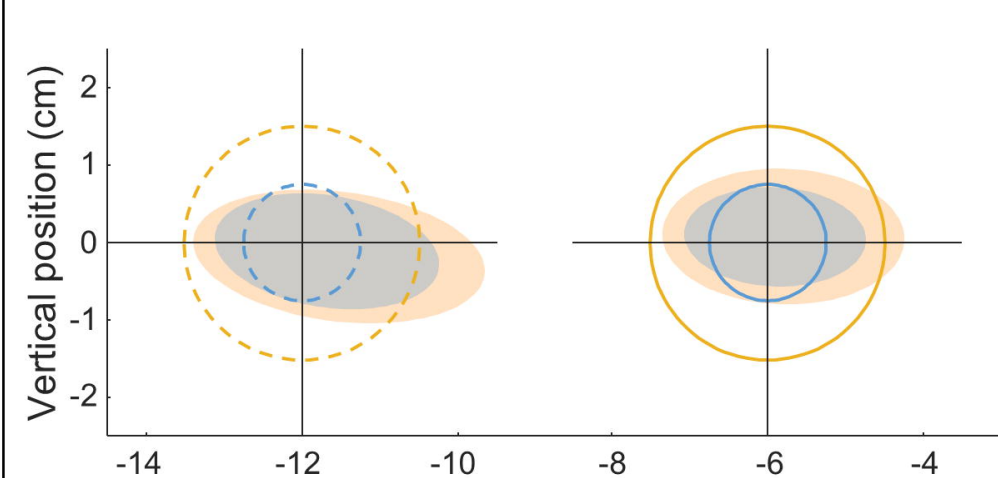

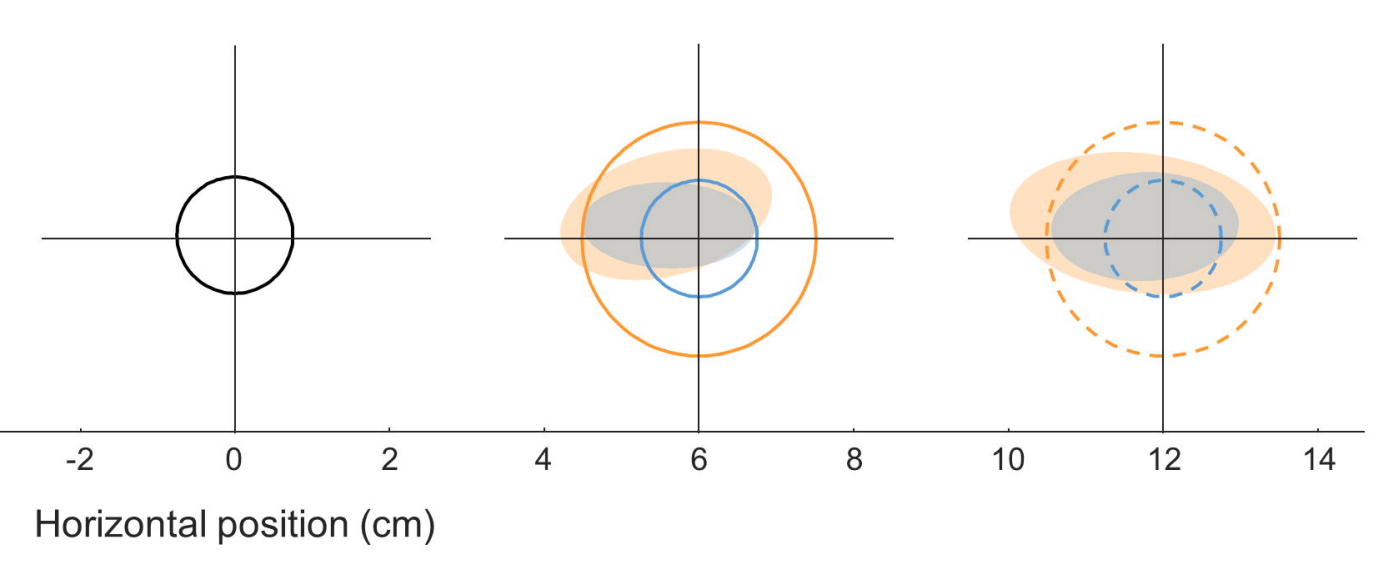

B

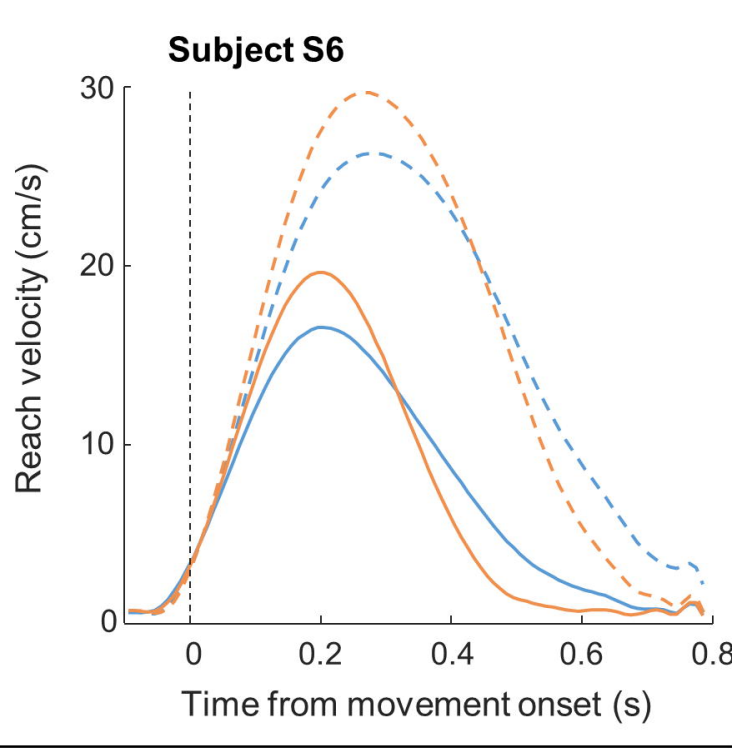




\section{Tokens task}

\section{A Size contrast}

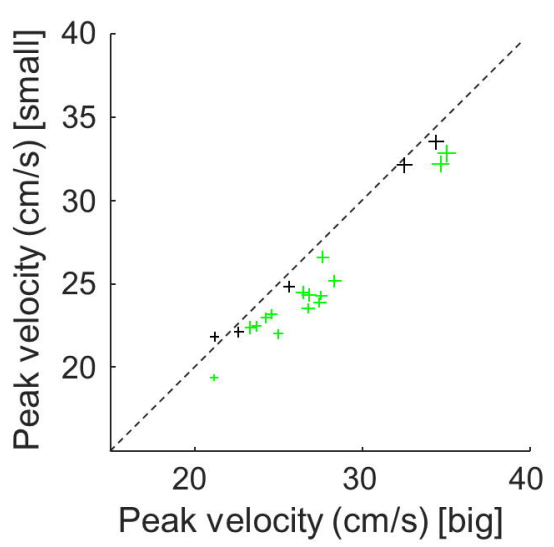

\section{B Distance contrast}

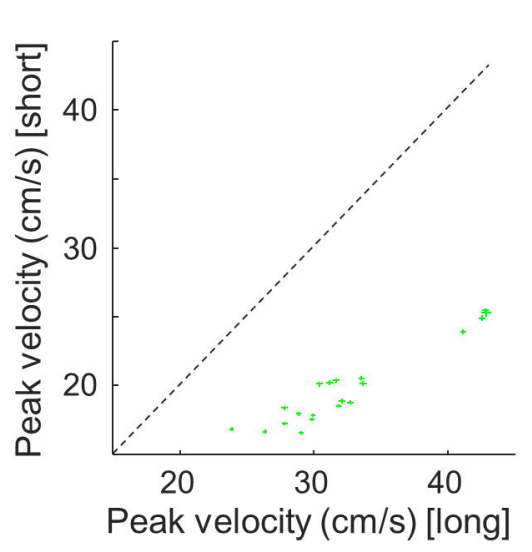

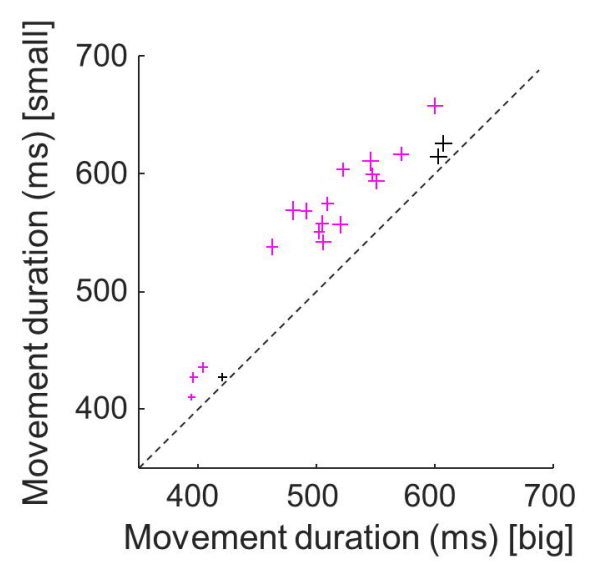

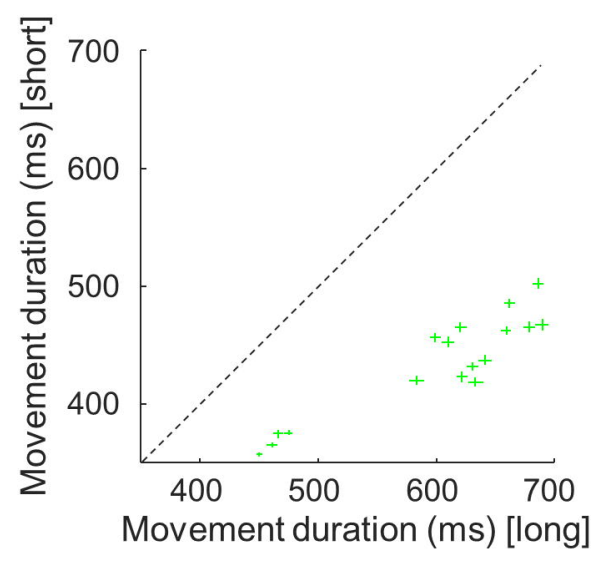

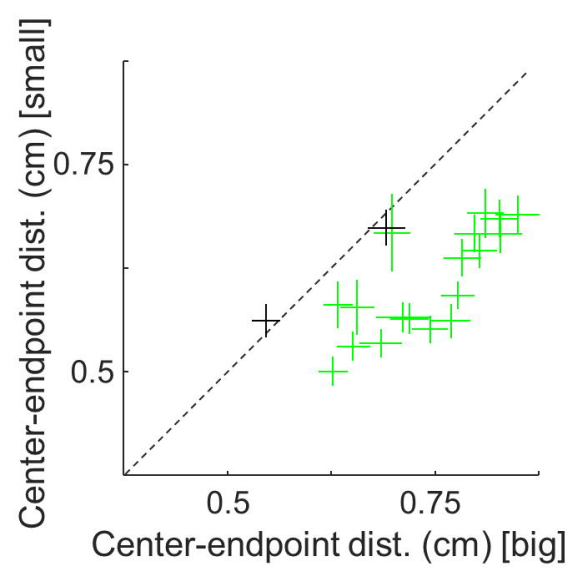

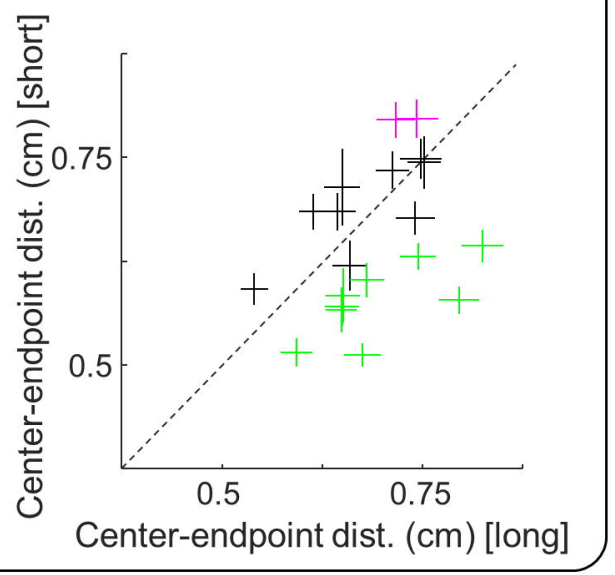




\section{Tokens task}

\section{A Size contrast}
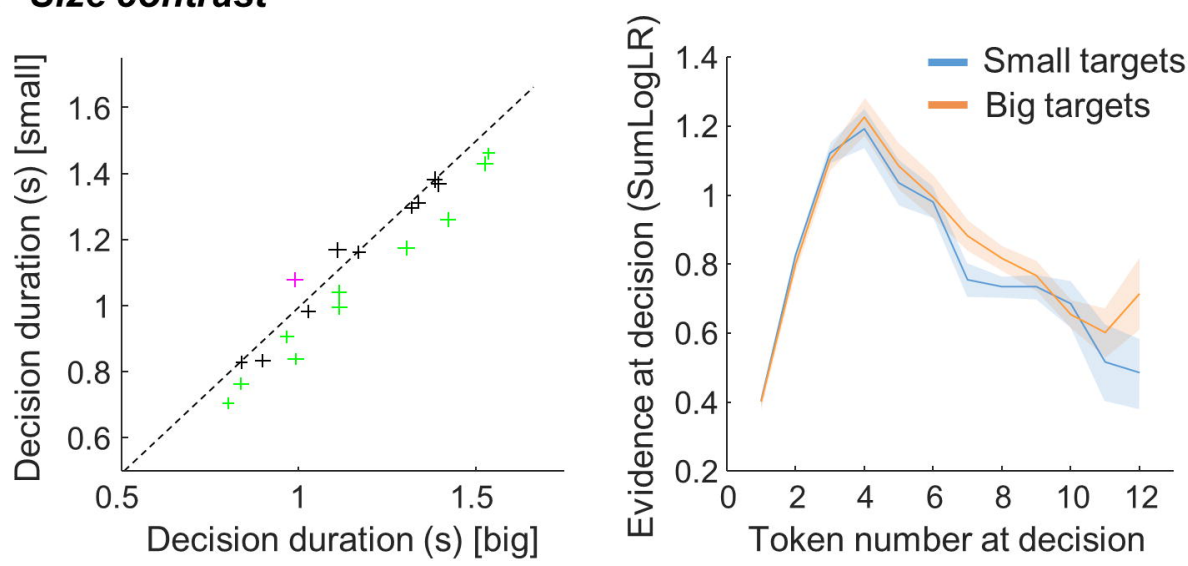

B Distance contrast
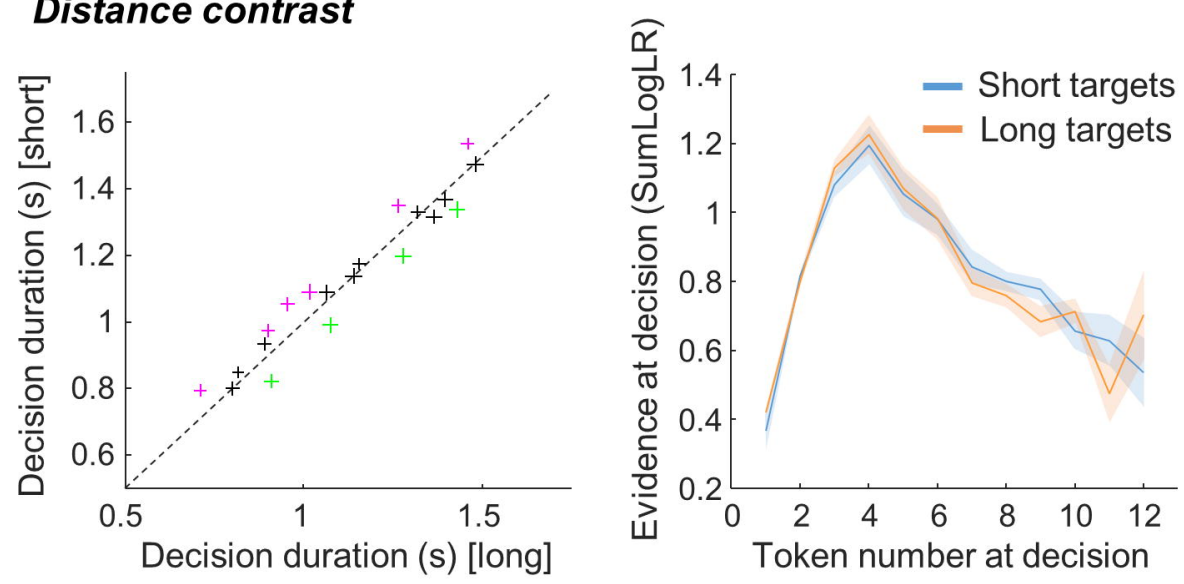
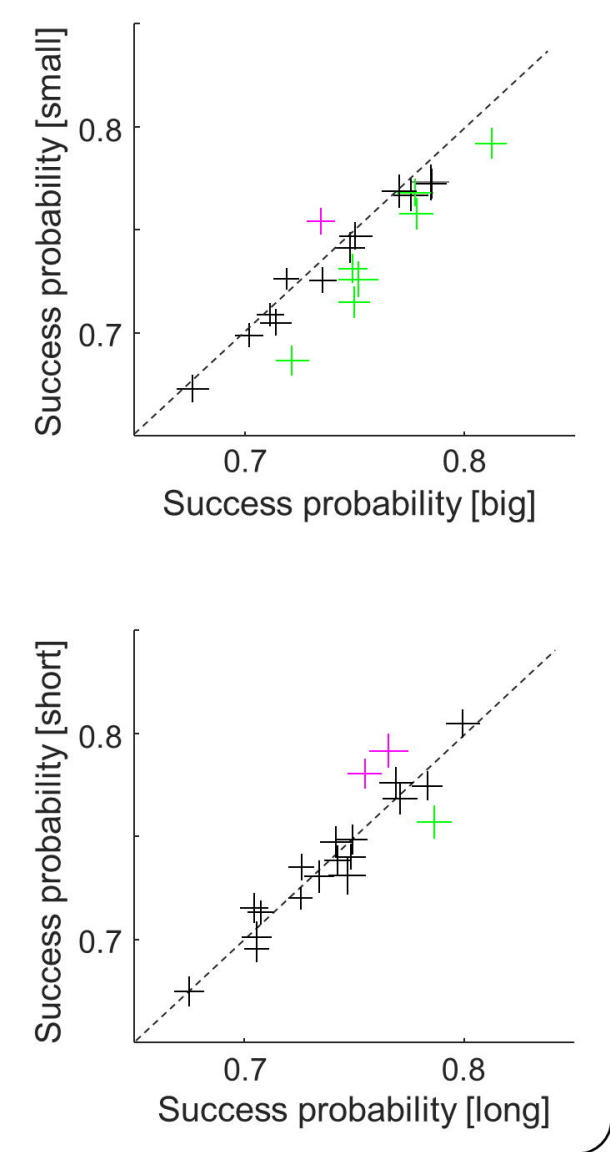

DR task

\section{Size contrast}

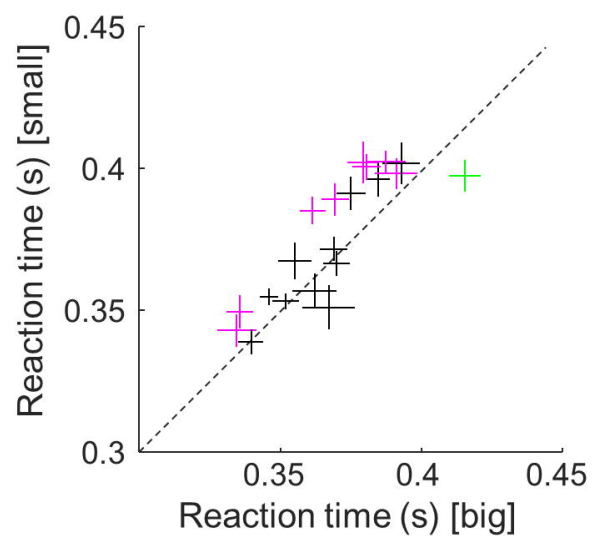

D Distance contrast

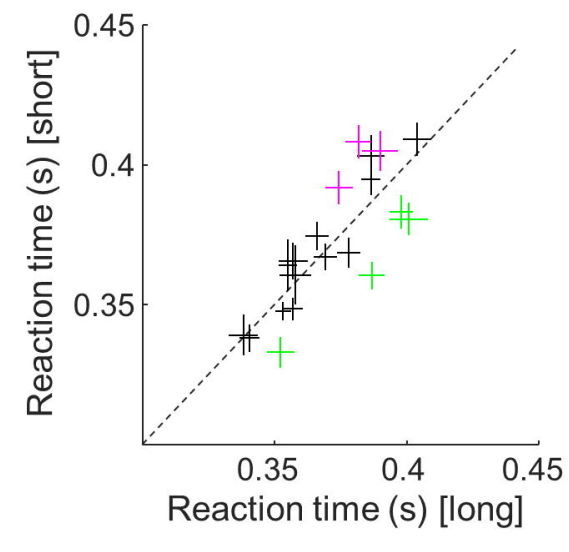


Size contrast

Distance contrast
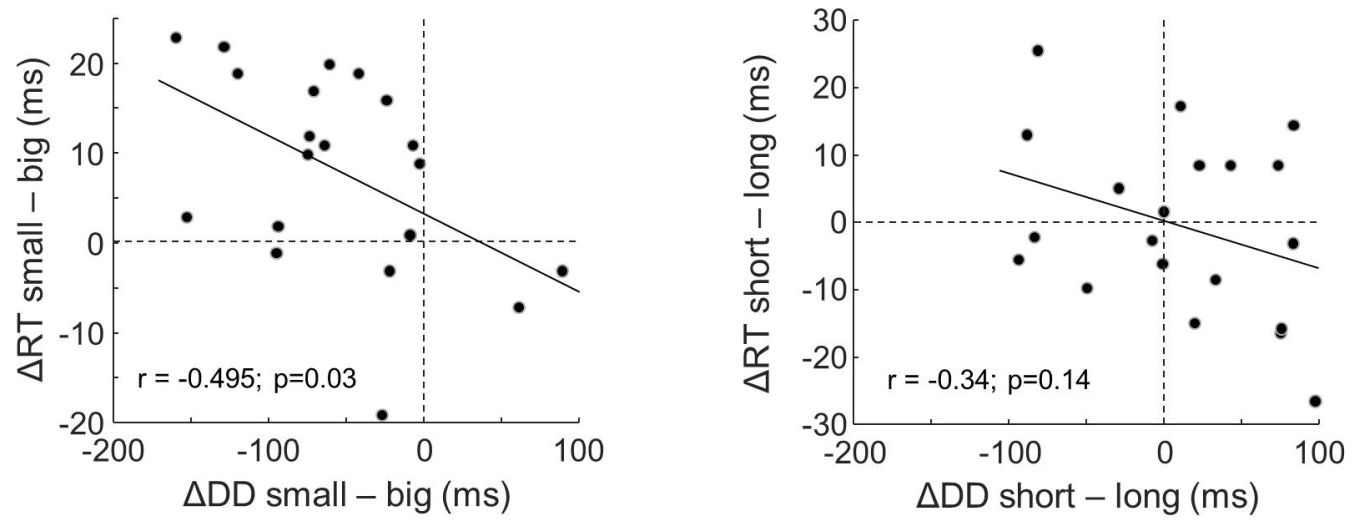


\section{A slow block}

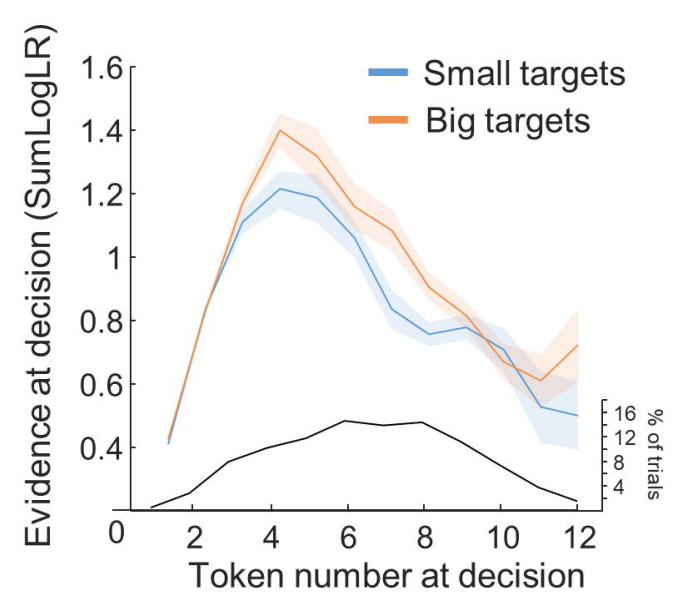

\section{B Fast block}

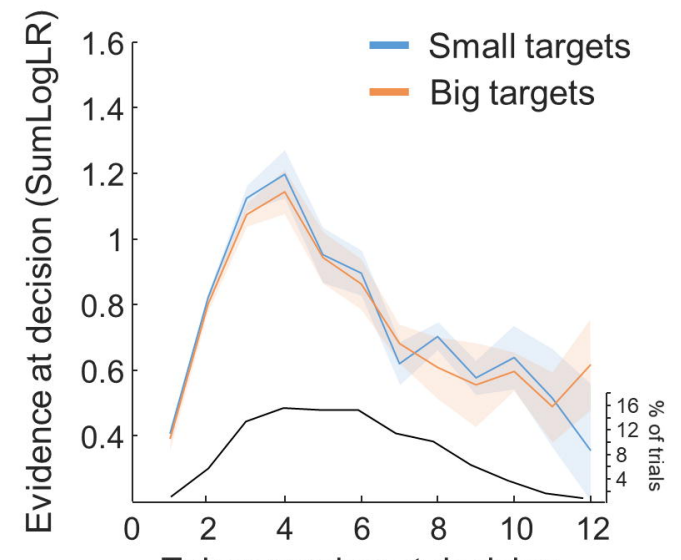

Token number at decision
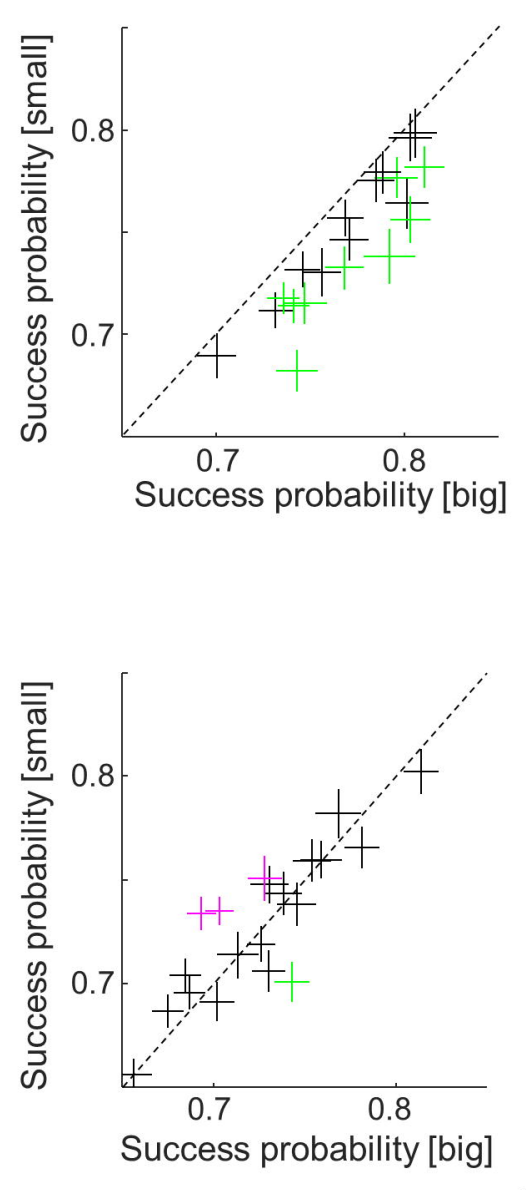

- Small targets

- Big targets

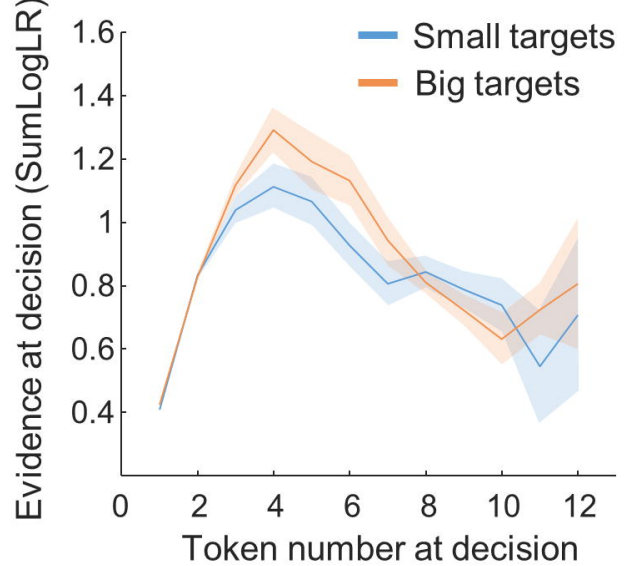

D Session \#2

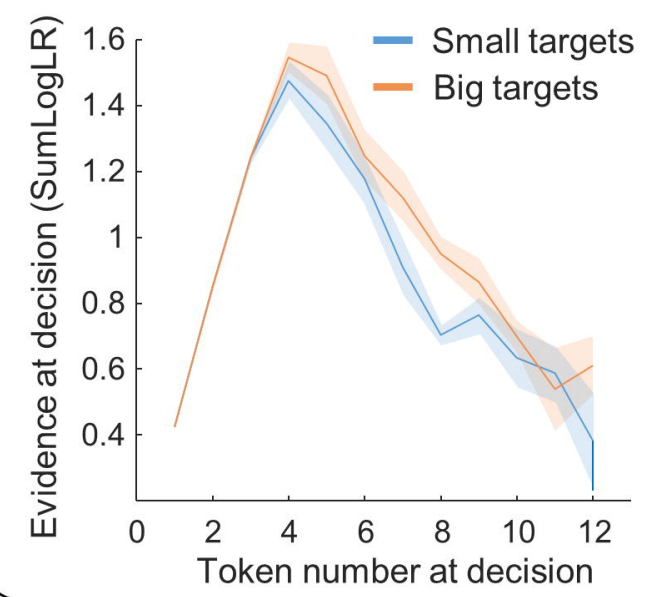

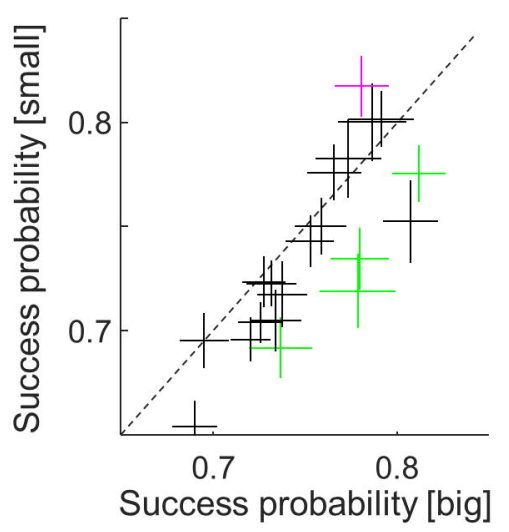

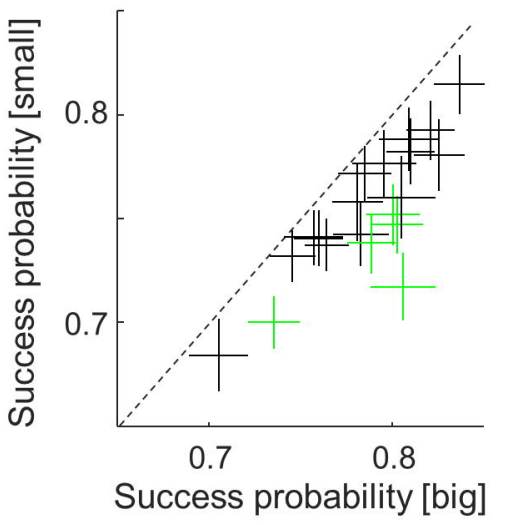



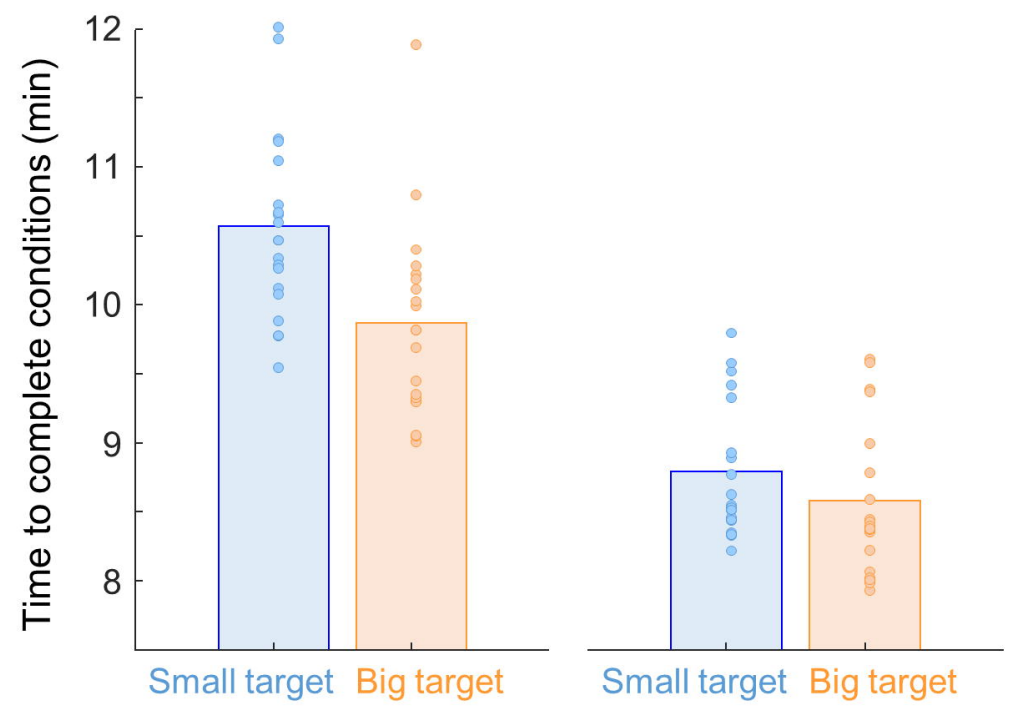